\title{
Effect of Segregated Alloying Elements on the High Strength Steel Properties: Application to the Large Size Ingot Casting Simulation
}

\author{
Chunping Zhang, Davood Shahriari, Abdelhalim Loucif, \\ Mohammad Jahazi, Louis-Philippe Lapierre-Boire \\ and Rami Tremblay
}

\begin{abstract}
Macrosegregation is one of the most significant defects which exert a determining effect on the properties of heavy ingots. The objective of this work is to study the influence of segregated solute elements on the physical and mechanical properties of a medium carbon high strength steel during large ingot casting process. The solidification process of a 20 Metric Tons (MT) ingot is simulated using Thercast FEM code. Different segregation levels of solute elements are picked up from a section along the longitudinal axis in the top of the ingot. Input steel data, including physical and mechanical properties, are determined by means of

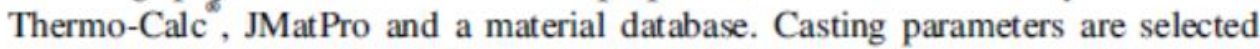
according to actual industrial operational conditions used for casting of large size ingots. Thermic and thermomechanic simulations are employed for calculating the solidification time. Results are analyzed in the framework of diffusion controlled solidification theory and the influence of alloying elements.
\end{abstract}

C. Zhang (®) - D. Shahriari - A. Loucif - M. Jahazi

École de Technologie Supérieur, 1100 Rue Notre-Dame Ouest,

Montréal, QUÉBEC H3C 1K3, Canada

e-mail: chunping.zhang.1@ens.etsmtl.ca

D. Shahriari

e-mail: Davood.Shahriari@etsmtl.ca

A. Loucif

e-mail: abdelhalim.loucif.1@ens.etsmtl.ca

M. Jahazi

e-mail: Mohammad.Jahazi@etsmtl.ca

L.-P. Lapierre-Boire - R. Tremblay

Finkl-Steel-Sorel, 100 Rue McCarthy, Saint-Joseph-de-Sorel, QUÉBEC

J3R 3M8, Canada

e-mail: lplapierre@ finkl.com

R. Tremblay

e-mail: rtremblay@finkl.com

() The Minerals, Metals \& Materials Society 2017

491

The Minerals, Metals \& Materials Society, TMS 2017 146th Annual Meeting \& Exhibition Supplemental Proceedings, The Minerals, Metals \& Materials Series, DOI 10.1007/978-3-319-51493-2_47

Authors' accepted manuscript, article published in TMS 2017 146th Annual Meeting \& Exhibition Supplemental Proceedings p. 491-500 (2017) https://doi.org/10.1007/978-3-319-51493-2_47

The final publication is available at link.springer.com 
Effect of Segregated Alloying Elements on the High Strength Steel Properties: Application to the Large Size Ingot Casting Simulation

Chunping Zhang, Davood Shahriari, Abdelhalim Loucif, Mohammad Jahazi, Louis-Philippe Lapierre-Boire and Rami Tremblay

C. Zhang $(\bowtie) \cdot$ D. Shahriari $\cdot$ A. Loucif $\cdot$ M. Jahazi

École de Technologie Supérieur

1100 Rue Notre-Dame Ouest, Montréal, Québec, H3C 1K3, Canada

e-mail: chunping.zhang.1@ens.etsmtl.ca

Davood.Shahriari @etsmtl.ca

abdelhalim.loucif.1@ens.etsmtl.ca

Mohammad.Jahazi@etsmtl.ca

tel.: 5143968800-7868

L.P. Lapierre-Boire $\cdot$ R. Tremblay

Finkl-Steel-Sorel

100 Rue McCarthy, Saint-Joseph-de-Sorel, Québec, J3R 3M8, Canada

e-mail: 1plapierre@ finkl.com

rtremblay@finkl.com

Keywords: Medium-carbon steel, Large size ingot, Segregated alloying elements, Casting simulation

\begin{abstract}
Macrosegregation is one of the most significant defects which exert a determining effect on the properties of heavy ingots. The objective of this work is to study the influence of segregated solute elements on the physical and mechanical properties of a medium carbon high strength steel during large ingot casting process. The solidification process of a 20 Metric Tons (MT) ingot is simulated using Thercast ${ }^{\circledR}$ FEM code. Different segregation levels of solute elements are picked up from a section along the longitudinal axis in the top of the ingot. Input steel data, including physical and mechanical properties, are determined by means of Thermo-Calc ${ }^{\circledR}$, JMatPro and a material database. Casting parameters are selected according to actual industrial operational conditions used for casting of large size ingots. Thermic and thermomechanic simulations are employed for calculating the solidification time. Results are analyzed in the framework of diffusion controlled solidification theory and the influence of alloying elements.
\end{abstract}

\title{
1 Introduction
}

For the production of large size mono-block forgings of high quality multi-component special steels, such as the shaft rotor for steam turbine and the pressure vessels for nuclear reactor, ingot casting is the only method. Macrosegregation, as a compositional heterogeneity, is one of the most significant defects occurring during the solidification process. It influences the thermophysical properties, affects the solidification behavior of casting, deteriorates the microstructure and mechanical properties, proves difficult to remove by subsequent thermo-mechanical treatment, and has haunted the manufacturers over decades.

Macrosegregation can be controlled by optimization of casting metal composition, dimensions of the ingot and casting/solidification processes. Thus the impact of ingot size [1], mold temperature [2], pouring superheat [3], pouring velocity [4], cooling rate [5], and steel composition [6] on the severity of macrosegregation has been investigated extensively. In order to free from the timely and costly 'trial and error' approach, finite element method (FEM) has been used for the simulation of the casting and solidification process. Numerical methods, however, generally use the input data of the fixed initial nominal composition. In reality, the solidification behavior is not only the nominal composition and processing parameters dependent but also varies with individual segregation domains. This study, therefore, was initiated to explore the effect of different segregation levels of solute elements on the material physical and mechanical properties, the solidification and cooling behavior as well as the solidification time so as to approximate the actual solidification process in large size castings. It will be of experimental and industrial 
significance for the macrosegregation research, the production process design and optimization of ingot manufacturing efficiency.

For this, three-dimensional thermic and thermomechanic simulations of the solidification process of a 20MT steel ingot were performed using Thercast ${ }^{\circledR} 8.2$ version FEM code [7]. Casting parameters were selected according to the actual industrial operational conditions. All the needed data are seldom available in the literature, especially for the steels like the medium carbon low-alloyed multicomponent steel in study. It is known that even small variations in the composition may have a significant effect on the material physical and mechanical properties. Thus, the input steel data, including physical and mechanical properties, were determined by means of Thermo-Calc ${ }^{\circledR}$ and JMatPro. Modeling results were analyzed in the framework of diffusion controlled solidification theory and the influence of segregated solute alloying elements. It should be mentioned that mesh size optimization for computation cost minimization and sensitivity analysis of the results have been performed and its selection was based on the analysis results.

\section{Material and Methods}

A cylindrical steel ingot was cast in a cast-iron mold. The steel melt composition is listed in Table 1 . Molten steel was poured from the mold bottom at about $1570^{\circ} \mathrm{C}$ and a layer of exothermic powder was over laid on the melt top. A hot-top, made of cast iron and lined with refractory materials inside, was placed on the top of the steel ingot. After solidification and stripping out, the hot-top with $30 \mathrm{~cm}$ 's head of the ingot was transversely cut off for the study purpose. A plate with about $2.5 \mathrm{~cm}$ in thickness was sliced on one side of the axial plane, as shown in Fig. 1a. The slice was then sectioned into over 200 samples and faces in the centerline plane along the longitudinal axis of all the samples were subject to grinding, and then chemical mapping using the Thermo Scientific ARL ${ }^{\mathrm{TM}} 4460$ mass spectrometer. The chemical composition of specimen was obtained by averaging out 3 random spectrometer measurements. Segregation maps of different elements in the longitudinal section of the hot-top and $30 \mathrm{~cm}$ 's head of ingot were then reconstructed and macrosegregation patterns were identified.

Typical thermophysical data including the temperature dependence of the density, the specific heat capacity, and the phase transformation temperatures of selected segregated solute levels were determined by means of the computational thermodynamics program Thermo-Calc ${ }^{\mathrm{B}}$ classic version $\mathrm{R}$ with the TCS Fecontaining slag database [8]. Thermomechanical behaviors, such as yield stress, Young's modulus, strain hardening exponent and strain-rate sensibility coefficient and their variations as a function of temperature were developed with the help of JMatPro software, version 9.0 [9]. The simulations of solidification were performed in the finite element code Thercast $^{\circledR}$ by using an Arbitrary Lagrangian-Eulerian (ALE) formulation for computing the thermal convection in liquid pool and mushy zone, and Lagrangian for calculating the deformation in solid regions. The method is applied both to mold filling simulation, where it can provide accurate free surface description, and to the study of thermo-mechanical phenomena occurring in the subsequent cooling down of cast parts: prediction of distortions and stresses.

Table 1 Nominal chemical composition of the investigated steel and the four positions in the upper region of the ingot (Wt.\%)

\begin{tabular}{cccccccccc}
\hline & $\mathrm{C}$ & $\mathrm{Mn}$ & $\mathrm{P}$ & $\mathrm{S}$ & $\mathrm{Si}$ & $\mathrm{Ni}$ & $\mathrm{Cr}$ & $\mathrm{Mo}$ & $\mathrm{Cu}$ \\
\hline Nominal & 0.35 & 0.82 & 0.007 & 0.002 & 0.4 & 0.15 & 1.79 & 0.46 & 0.13 \\
1 & 0.33 & 0.82 & 0.01 & 0 & 0.39 & 0.17 & 1.83 & 0.39 & 0.13 \\
2 & 0.6 & 0.94 & 0.024 & 0.002 & 0.5 & 0.18 & 2.1 & 0.52 & 0.15 \\
3 & 0.37 & 0.86 & 0.013 & 0.001 & 0.4 & 0.17 & 1.92 & 0.44 & 0.14 \\
4 & 0.44 & 0.86 & 0.015 & 0.001 & 0.42 & 0.17 & 1.89 & 0.43 & 0.14 \\
\hline
\end{tabular}

\section{Results and Discussion}

Solute distribution along the centerline longitudinal axis of the ingot presents sort of symmetry which confirms the formation of type A macrosegregation. The concentrations of all the elements increase continuously from the ingot lower bordering part to the center upper region, attesting that the liquid-solid interface has set off from the mold wall and globally advanced inwards and upwards to the last region to solidify located at the top center due to the efficient thermal protection of the hot-top. Concentrations of most elements in both the hot-top and the head of the ingot are higher than the nominal one, which 
indicates that positive segregation has taken place in these regions. As seen in Fig. $2 \mathrm{~b}$, the concentration profiles of carbon and chromium along the lines at the top and the bottom of the section in study display all the above-mentioned characteristics. Four representative segregation levels, corresponding to the highest and lowest segregation severity along the ingot centerline and the line next to the mold, were picked up for the analysis. Their chemical compositions are provided in Table 1 also in order to facilitate the comparison with the nominal composition. Their positions in the section are labeled in Fig. 1a. The numbers given to the four positions are kept consistent hereinafter. The segregation severity level of solute elements in the four studied sites can be sequenced in descending order as $2>4>3>1$.

\subsection{Solidification Model Description}

Considering continuum medium as illustrated in Fig. 2, different behaviors of the metal are distinguished by the critical temperature $\mathrm{T}_{\mathrm{c}}$, above which a thermo-viscoplastic behavior is considered and below which a thermo-elasto-viscoplastic one [7]. In order to take into account the complex behavior of solidifying alloys, a hybrid constitutive mold was used. In the liquid state, the metal was treated as a Newtonian fluid and the Navier-Stokes equation with temperature-dependent terms were employed. When the metal begins to solidify, the alloy in the mushy state was modeled as a non-Newtonian fluid obeying a Thermo-viscoplastic Norton-Hoff law. In the calculation of the deformation in solid regions using Lagrangian formulation, the computational grid was allowed to move with the material: this was essential to treat the air gap between mold and the casting. The liquid contraction and the solidification shrinkage were taken into consideration when the ALE scheme was applied to compute the thermal convection in liquid pool and mushy zone so as to prevent the mesh from degenerating and allow tracking the free surfaces.

3D linear tetrahedral elements were used for the discretization of the part and mold components. The unilateral contact condition (loss of contact, that is separation, is accepted) was applied to the boundary between mold and casting. In the resolution stage, a coupled computation of the stress and temperature fields was performed so that equations were solved iteratively using a prescribed time step. Due to the symmetry conditions of the casting system, only one quarter of the 20MT ingot was modelled, the configuration of which is displayed in Fig. 3. The mold was considered as non-deformable. The finite element mesh of the solidification system consisted of 1,190,000 elements which were selected based on several mesh refinement studies.

\subsection{Effect of Segregation Levels on Physical Properties}

Different segregation levels result in the change of the kinetics of phase transformations, and then the difference in thermophysical and mechanical properties related to the phases formed. Figs. $4 \mathrm{a}$ and $4 \mathrm{~b}$ show the evolution of solid fraction and density with the temperature for the four selected segregated compositions, respectively. As mentioned above, the segregation severity for the four representative positions in descending order is $2>4>3>1$. It can be seen from Fig. 4 that the evolution of physical properties is in reverse order to the segregation severity level. Lower segregation results in the progressive augmentation of phase transformation temperature, including the solidus, liquidus and the transformation point from delta ferrite $(\delta)$ to austenite $(\gamma)$. In the highest segregation level, the position 2 , no $\delta-\gamma$ transformation took place. In addition, lower segregation levels induce smaller temperature differences between the solidus and liquidus, corresponding to slightly higher element liquid/solid partition coefficient. The observed increased density in lower segregation level may be attributed to more alloying elements dissolved. Such tendency in the density evolution agrees well with the experimental analysis results which indicated low concentration of small porosities in the ingot's center upper region.

The difference in segregation levels, in the meantime, brings about the discrepancy in elastic and plastic behaviors, as observed in Fig. 5. The evolution of the liquid viscosity displays the same decreasing trend with the increase of the segregation severity. The reported deterioration effect of higher content of alloying elements on toughness and plasticity is in accordance with the calculation results [10]. The reduction of density with the increased severity level of segregation may also be the reason. The solute diffusion coefficient in liquid is discovered, based on Stokes-Einstein equation [11], inversely proportional to solvent viscosity. Thus, the diffusion coefficients in liquid in the four positions can be sequenced as D2 $>$ D4 $>$ D3 $>$ D1, in agreement with the segregation severity descending order. All these variations in properties due to different segregation levels affect the heat transfer, convection and radiation modes, as 
well as stress and deformation domain during mold filling, cooling down and the progressive solidification of the ingot.

\subsection{Effect of Segregation Levels on Solidification Time and Temperature Gradient}

The knowledge of solidification time of a large size ingot in the industry is of quite importance in that shorter solidification time will not allow achieving solid state at the center of the ingot and longer time will result in increased segregation levels and porosities. Therefore, solidification time is taken as the simulation result to discuss. Solidification times needed for the four analyzed positions in the casting are different and vary with the segregation severity level. Specifically, solidification time at the center is longer than the one in the mold side, as presented in Fig. 6. It should be noted that for presentation purposes, the simulation was stopped for a maximum temperature of $1000^{\circ} \mathrm{C}$ to make sure that solid state is achieved at the center of the large size ingot. The observed evolution of solidification time with the percent carbon was approximated with a second degree polynomial function and is illustrated in Fig. 6, where $y$ is the solidification time and $x$ carbon weight composition. As indicated in the figure, the solidification time attains its peak value at $0.513 \mathrm{wt} . \%$ carbon, corresponding to the composition located in the interface between the ingot and the hot-top, which probably concerns the pipe formation and the time for its creation.

Figs. 7 and 8 illustrate respectively the thermic and thermomechanic simulations of the cooling of the cast part with the composition of the position 4 until the solidus temperature, $1414^{\circ} \mathrm{C}$, is reached. It can be seen that the solidification time decreases around 25 minutes, passing from about 8 hours 48 minutes to 8 hours 23 minutes or so when the influence of the solid deformation is taken into account. According to the simulation results, although the last region to solidify is always located at the top center of the hot-top, solid mechanical deformation gives rise to the alteration of thermal gradient, particularly in the interior of the ingot. The free surface can be seen almost flat in Fig. 8 because of an efficient thermal protection which minimizes the thermal gradient in the hot-top.

\section{Conclusion}

This research presented a study on the effect of different segregation levels on the solidification behavior of a 20MT steel ingot. Comparison analyses of the physical and mechanical properties of representative segregation levels were performed by means of softwares like Thermo-Calc ${ }^{\circledR}$ and JMatPro. Solidification time of varied segregation severities was calculated through thermic and thermomechanic simulations of the mold filling and cooling behavior of steel ingot using Thercast ${ }^{\circledR}$ FEM code. The following main conclusions can be made from this study:

1. The evolution of physical properties and elastic/plastic behaviors is in reverse order to the segregation severity level due to different solute diffusion coefficient in liquid and higher content of alloying elements.

2. By increasing the segregation severity level, solidification time increases. Solid mechanical deformation gives rise to the alteration of thermal gradient and the decrease of solidification time.

\section{Acknowledgements}

The financial support from the Natural Sciences and Engineering Research Council (NSERC) of Canada in the form of a Collaborative Research and Development Grant (CRDG) under number 470174 is gratefully acknowledged. Finkl Steel-Sorel Co. for providing the material and Transvalor Americas Corp. for permission and technical support on Thercast software are greatly appreciated.

\section{References}

1. D.R. Liu et al., "Modeling of Macrosegregation in Steel Ingot: Influence of Mold Shape and Melt Superheat," Kovove Mater, 19 (2011), 1-11 
2. Y. Zhang et al., "Numerical Simulation and Optimization in Solidification of Zinc Alloy," Advanced Materials Research, 287-290 (2011), 2902-2905

3. T. Pikkarainen et al., "Effect of Superheat on Macrostructure and Macrosegregation in Continuous Cast Low-alloy Steel Slabs," $4^{\text {th }}$ International Conference on Advances in Solidification Processes, IOP Conf. Series: Materials Science and Engineering, 117 (2016), 012064

4. J. Zeng, and W. Chen, "Effect of Casting Speed on Solidification Structure and Central Macrosegregation during Continuous Casting of High-carbon Rectangular Billet," La Metallurgia Italiana, 7/8 (2015), 51-58

5. P.G. Xu, F. Yin, and K. Nagai, "Solidification Cooling Rate and As-cast Textures of Low-carbon Steel Strips," Materials Science and Engineering A, 441 (2006), 157-166

6. D. Shahriari et al., "3D Numerical Simulation of Solidification of Large Size Ingots of High Strength Steel," SteelSim 2015. $6^{\text {th }}$ International Conference on Modelling and Simulation of Metallurgical Processes in Steelmaking.

7. Thercast User Manual, (France: Transvalor, 2012)

8. ThermoCalc Software AB, (Stockholm Technology Park, 2006)

9. JMatPro User's Guide (Sente Software Ltd., 2005)

10. J. He, "21 Chemical Elements and Effects on Steel Mechanical Properties," (Report of OTAI SpecialSteel Co. Ltd., 2015)

11. C. Miller, "The Stokes-Einstein Law for Diffusion in Solution," Proceedings of the Royal Society of London, Series A, Containing Papers of a Mathematical and Physical Character, 106 (740) (1924) 724749

\section{Figure captions:}

Fig. 1 a Cutting diagram of the ingot for chemical analysis and the four analyzed positions, b Concentration profiles of carbon and chromium along the lines at the top and bottom of the sectioned slice

Fig. 2 Material behaviors depending on its condition in the process of solidification [7]

Fig. 3 a Geometry of the cast part, b Finite element mesh of the casting and of the mold

Fig. 4 Temperature dependence of physical properties for different segregation levels

Fig. 5 Evolution of mechanical properties with segregation severity levels

Fig. 6 Plot of solidificaiton time as a function of weight percentage of carbon

Fig. 7 Thermic simulation: a Temperature distribution, b Solidification time after 100\% solidification of position 4

Fig. 8 Thermomecanic simulation: a Temperature distribution, b Solidification time after 100\% solidification of position 4 
a

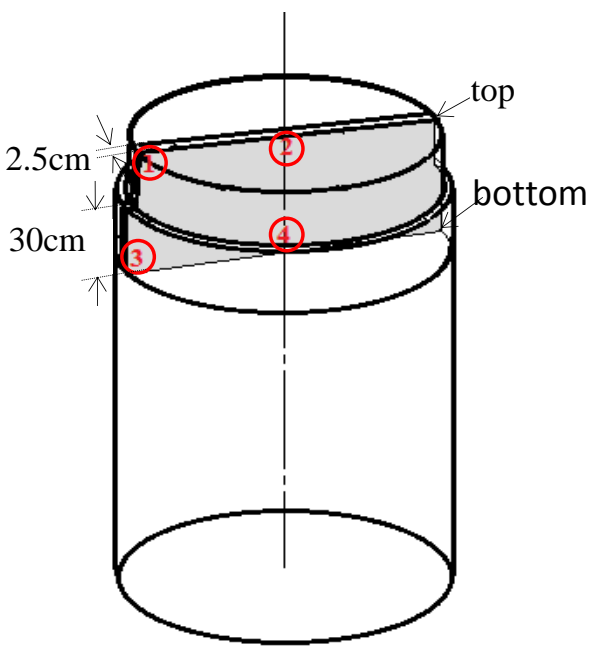

b

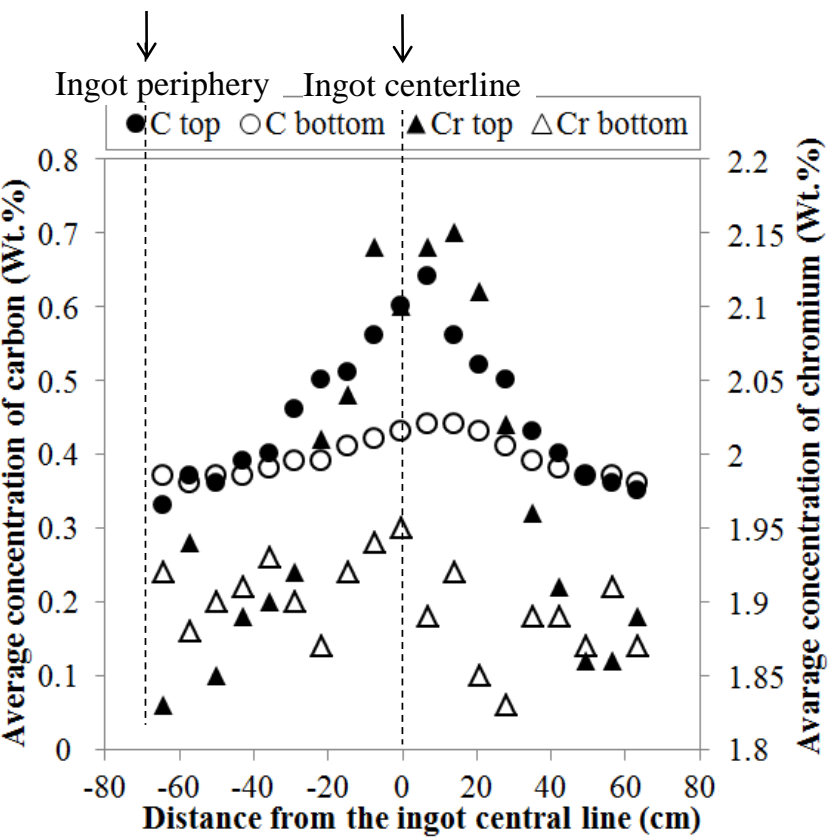

Fig. 1 a Cutting diagram of the ingot for chemical analysis and the four analyzed positions, b Concentration profiles of carbon and chromium along the lines at the top and bottom of the sectioned slice

\begin{tabular}{|c|c|c|c|}
\hline \multirow[b]{2}{*}{ Liquid } & Solid $\mathrm{fl}_{\mathrm{l}}$ & 1 & \multirow[b]{2}{*}{ Solid } \\
\hline & Semi-liquid & Semi-solid & \\
\hline \multicolumn{2}{|c|}{$\begin{array}{c}\uparrow \\
\mathrm{T}_{\text {liquidus }}\end{array}$} & \multicolumn{2}{|c|}{$\begin{array}{c}\uparrow \\
\mathrm{T}_{\text {solidus }}\end{array}$} \\
\hline Newtonian & Thermo-visco-plastic & Elasto-visco-plastic & Elasto-plastic \\
\hline Navier-Stokes & Northon-Hoff & Perzyna & \\
\hline
\end{tabular}

Fig. 2 Material behaviors depending on its condition in the process of solidification [7]

Fig. 3 a Geometry of the cast part, b Finite element mesh of the casting and of the mold $\mathbf{a}$

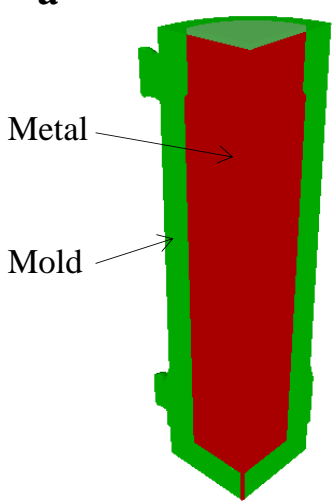

b

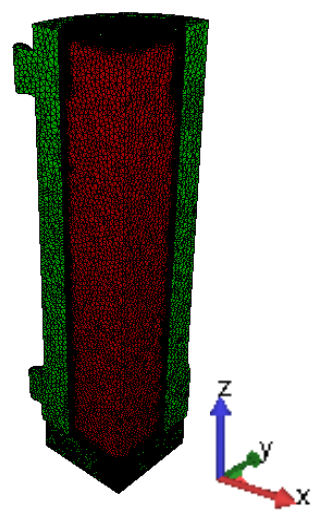



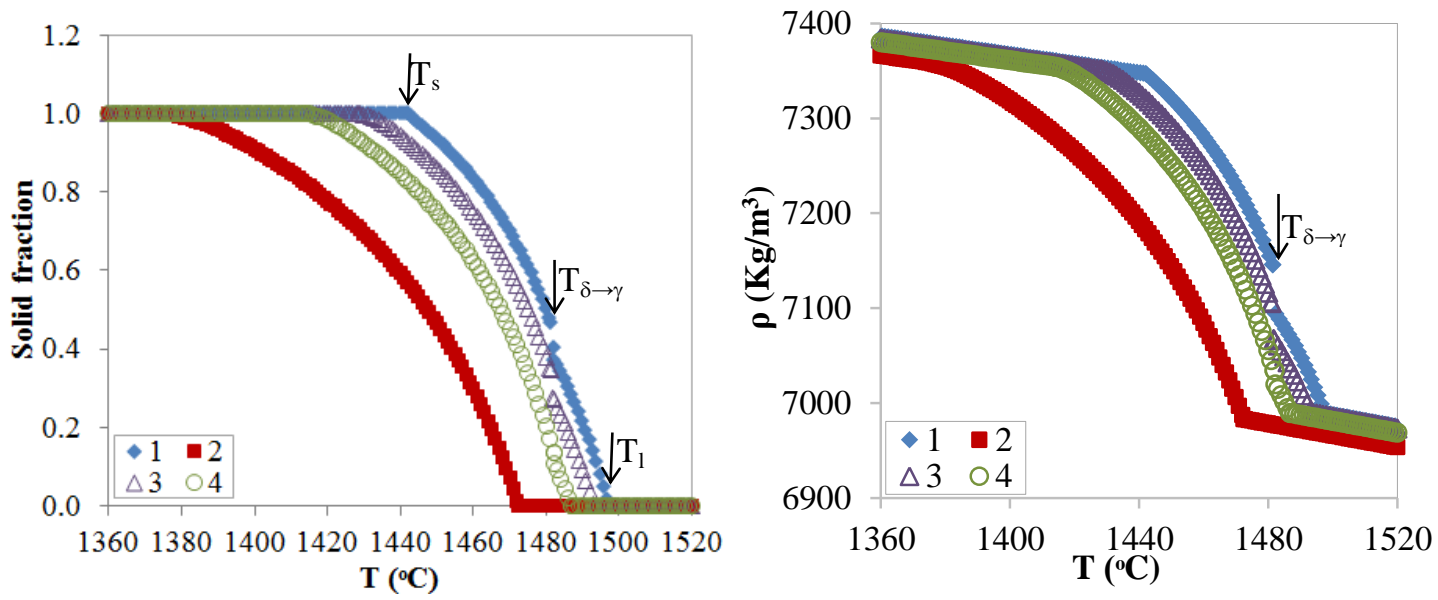

Fig. 4 Temperature dependence of physical properties for different segregation levels
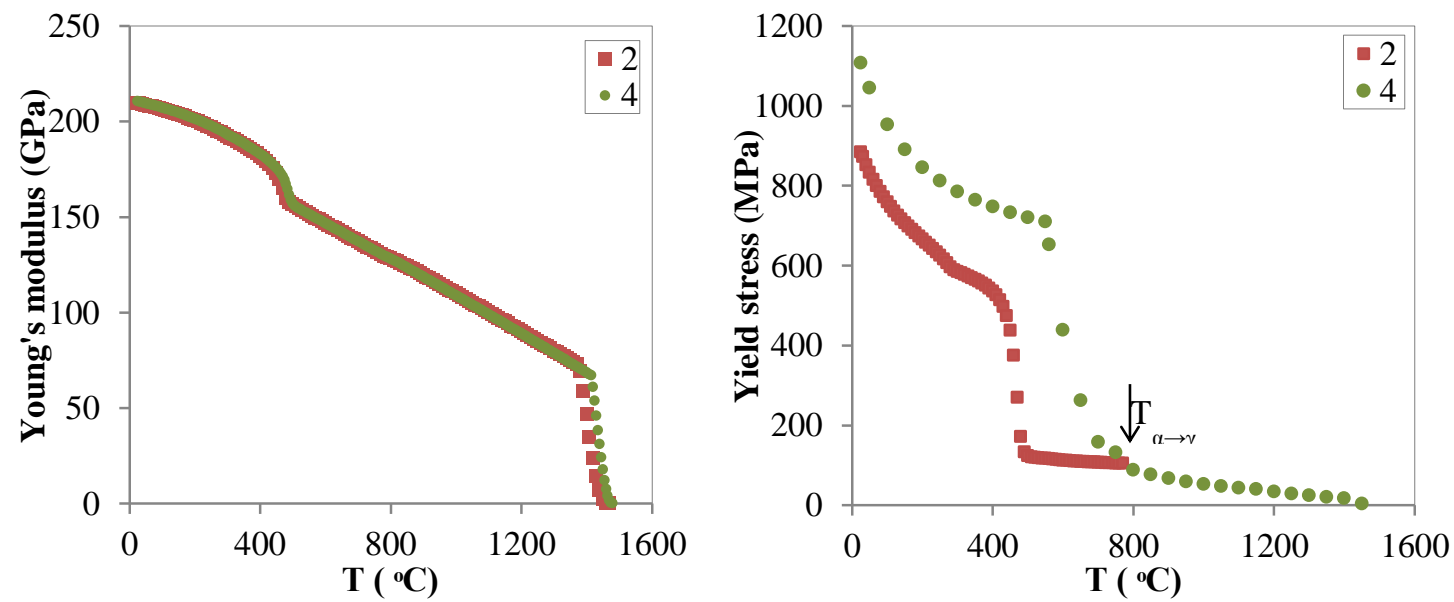

Fig. 5 Evolution of mechanical properties with segregation severity levels

Fig. 6 Plot of solidification time as a function of weight percentage of carbon

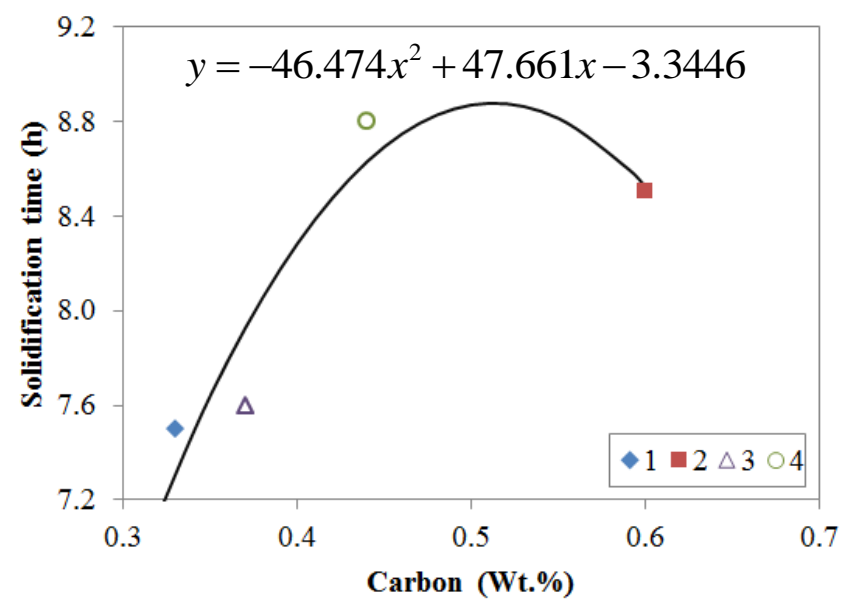


Fig. 7 Thermic simulation: a Temperature distribution, b Solidification time after $100 \%$ solidification of position 4 $\mathbf{a}$

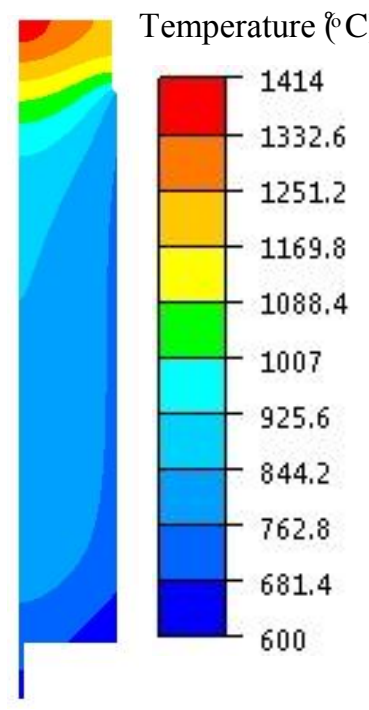

a

Fig. 8 Thermomechanic simulation: a Temperature distribution, b Solidification time after $100 \%$ solidification of position 4

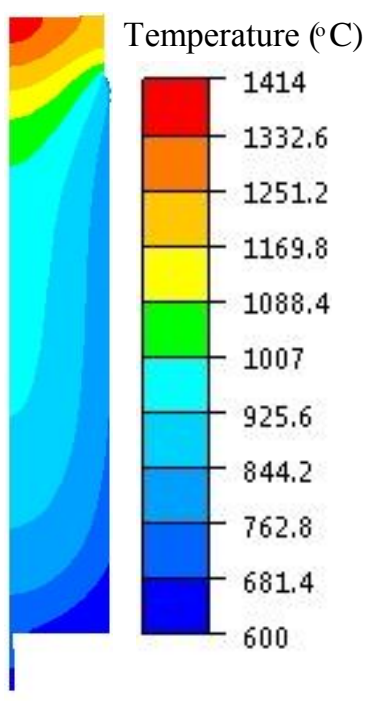

b

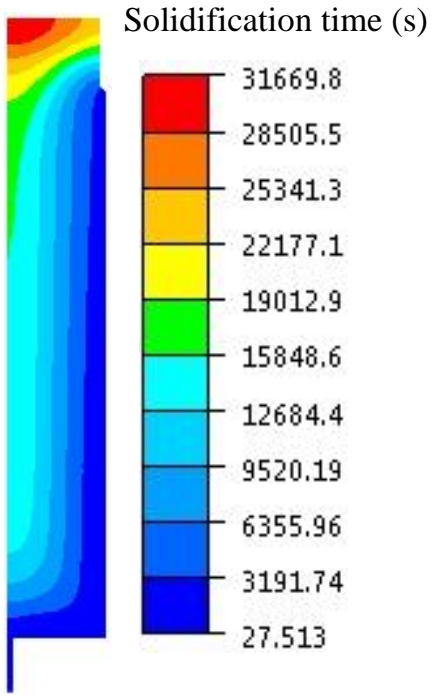

b

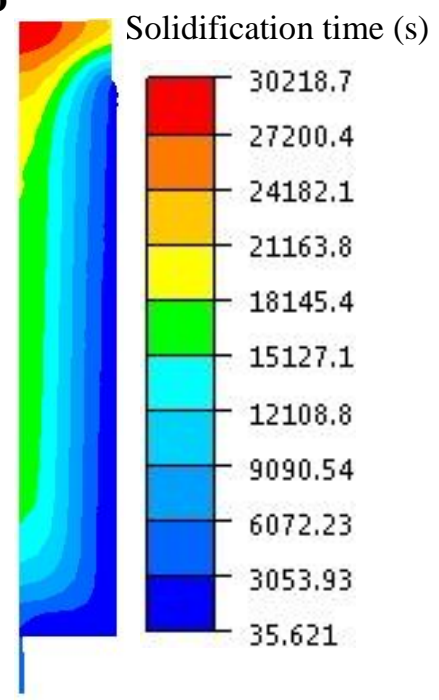

